\title{
Energy in higher-derivative gravity via topological regularization
}

\author{
Gaston Giribet \\ Center for Cosmology and Particle Physics, New York University, \\ 726 Broadway, New York City, New York 10003, USA \\ Olivera Miskovic ${ }^{\dagger}$ \\ Instituto de Física, Pontificia Universidad Católica de Valparaíso, Casilla 4059, Valparaiso, Chile \\ Rodrigo Olea ${ }^{\ddagger}$ and David Rivera-Betancour ${ }^{\S}$ \\ Departamento de Ciencias Físicas, Universidad Andres Bello, Sazié 2212, Piso 7, Santiago, Chile
}

(Received 2 July 2018; published 27 August 2018)

\begin{abstract}
We give a novel definition of gravitational energy for an arbitrary theory of gravity including quadraticcurvature corrections to Einstein's equations. We focus on the theory in four dimensions, in the presence of a negative cosmological constant, and with asymptotically anti-de Sitter (AdS) boundary conditions. As a first example, we compute the gravitational energy and angular momentum of Schwarzschild-AdS black holes, for which we obtain results consistent with previous computations performed using different methods. However, our method is qualitatively different due to the fact that it is intrinsically nonlinear. It relies on the idea of adding to the gravity action topological invariant terms which suffice to regularize the Noether charges and render the variational problem well-posed. This is an idea that has been previously considered in the case of second-order theories, such as general relativity and which, as shown here, extends to higher-derivative theories. Besides black holes, we consider other solutions such as gravitational waves in AdS, for which we also find results that are in agreement. This enables us to investigate the consistency of this approach in the non-Einstein sector of the theory.
\end{abstract}

DOI: 10.1103/PhysRevD.98.044046

\section{INTRODUCTION}

Topology plays a fundamental role in theoretical physics, from the theory of fundamental particles to the study of topological phases of matter. It is a central notion in the study of dynamical systems and, from there, it extends to practically every area of physics. Derived notions-such as topological classes and topological invariants-are essential tools in gauge field theories and quantum gravity, being indispensable for the study of anomalies, quantum transitions, and topological defects. In the case of the theory of gravity, being a theory of the geometry of the spacetime itself, the role played by topological invariants is particularly important. They appear in relation to the Euclidean path-integral formulation of the theory, in the study of

\footnotetext{
*gg1043@nyu.edu

olivera.miskovic@pucv.cl

${ }^{\ddagger}$ rodrigo.olea@unab.cl

§d.riverabe@gmail.com
}

Published by the American Physical Society under the terms of the Creative Commons Attribution 4.0 International license. Further distribution of this work must maintain attribution to the author(s) and the published article's title, journal citation, and DOI. Funded by SCOAP ${ }^{3}$. gravitational instantons, and in higher-dimensional, highercurvature extensions of the theory. Here, following an idea previously explored by one of the authors and collaborators in the case of second-order theories such as general relativity [1], we propose a novel use of topological invariants in the context of gravity, namely, as regulators of the Noether-charge computation in theories that include higher-derivative terms. This is a very important problem, as higher-derivative corrections to Einstein's theory naturally arise when quantum effects are taken into account, and therefore having a method to compute the observables in those cases is crucial. For theories of this sort, we show that the Euler characteristic - which in virtue of the ChernWeil-Gauss-Bonnet theorem is expressed in terms of the Riemann tensor - can be used to regularize the infrared divergences that typically appear when trying to compute the conserved Noether charges in asymptotically, locally maximally symmetric spaces. Such divergences arise due to the noncompactness of the spacetime but, as we will show, the inclusion of the topological invariant in the action suffices to render the result finite and the variational problem well-posed. We refer to this phenomenon as topological regularization. As said, this has been observed to occur in theories with second-order field equations, such as general relativity (GR). Here, we show that this is, 
indeed, a much more general phenomenon, which also occurs in higher-derivative theories with arbitrary quadratic corrections to the Einstein-Hilbert action. Having such a nonlinear method to compute charges in theories with actions that are quadratic in the curvature tensor and in the presence of a cosmological constant is very important, as such theories are known to suffer from linear instabilities that cause the linear analysis to break down.

\section{QUADRATIC-CURVATURE GRAVITY}

Quantum effects induce higher-derivative corrections to the effective gravitational action. This is particularly realized in string theory, where such corrections can be computed explicitly to different orders. In the case of heterotic string theory, for example, $R^{2}$ corrections are seen to appear at the next-to-leading order in the $\alpha^{\prime}$ expansion of the low-energy effective action. $R^{2}$ terms also arise in different string theory compactifications. Here, aiming at not restricting ourselves to any specific model, we will consider the most general quadratic-curvature gravity (QCG) theory in four dimensions and in the presence of a cosmological constant. This is described by the action $I=(16 \pi G)^{-1} \int d^{4} x \sqrt{-g} \mathcal{L}$ with the Lagrangian density given by an arbitrary combination of Ricci-squared and scalar curvature-squared terms, namely,

$$
\mathcal{L}=R-2 \Lambda+\alpha R^{\mu \nu} R_{\mu \nu}+\beta R^{2} .
$$

The cosmological constant $\Lambda$ (which here we consider to be negative) is related to the radius $\ell$ of the anti-de Sitter (AdS) solution by $\Lambda=-3 / \ell^{2}$. In Eq. (1), $\alpha$ and $\beta$ are two arbitrary coupling constants of mass dimension -2 , which set the length scale $l=\max \{\sqrt{|\alpha|}, \sqrt{|\beta|}\}$ at which the higher-curvature effects start to dominate over the EinsteinHilbert term. The action (1) does not include the Kretschmann scalar (Riemann-squared term), as in four dimensions its presence can always be compensated by a redefinition of the couplings $\alpha$ and $\beta$, up to a boundary term.

As already mentioned, nonlinear terms in the Riemann tensor are expected to appear in the quantum gravity effective action. The presence of such terms improves the ultraviolet behavior of the theory relative to GR. In fact, the theory defined by the Lagrangian (1) turns out to be renormalizable [2]. The price to be paid for this is the emergence of ghost-like additional degrees of freedom. The class of theories given by the Lagrangian (1) leads to fourth-order field equations which generically describe a massive scalar mode and a massive spin-2 field, in addition to the massless graviton already present in GR.

Taking arbitrary variations of the action yields the equation of motion (EOM) of the theory plus a surface term,

$$
\delta I=\frac{1}{16 \pi G} \int_{M} d^{4} x \sqrt{-g}\left(E^{\mu \nu} \delta g_{\mu \nu}+\nabla_{\alpha} \Theta^{\alpha}\right),
$$

where the field equations are given by $E^{\mu \nu}=0$, with the symmetric rank-2 tensor $E^{\mu \nu}=G^{\mu \nu}+\alpha E_{(\alpha)}^{\mu \nu}+\beta E_{(\beta)}^{\mu \nu}$ being composed of the Einstein tensor

$$
G^{\mu \nu}=R^{\mu \nu}-\frac{1}{2} R g^{\mu \nu}+\Lambda g^{\mu \nu}
$$

supplemented with the two contributions coming from the quadratic-curvature terms,

$$
\begin{aligned}
E_{(\alpha)}^{\mu \nu}= & \left(g^{\mu \nu} \square-\nabla^{\mu} \nabla^{\nu}\right) R+\square\left(R^{\mu \nu}-\frac{1}{2} g^{\mu \nu} R\right) \\
& +2\left(R^{\mu \sigma \nu \rho}-\frac{1}{4} g^{\mu \nu} R^{\sigma \rho}\right) R_{\sigma \rho}
\end{aligned}
$$

and

$$
E_{(\beta)}^{\mu \nu}=2 R\left(R^{\mu \nu}-\frac{1}{4} g^{\mu \nu} R\right)+2\left(g^{\mu \nu} \square-\nabla^{\mu} \nabla^{\nu}\right) R .
$$

For a generic gravity theory, surface terms can be found as partial derivatives of the Lagrangian with respect to the Riemann tensor in the form of

$$
\Theta^{\alpha}(\delta g, \delta \Gamma)=2 E_{\mu \nu}^{\alpha \beta} g^{\nu \lambda} \delta \Gamma_{\beta \lambda}^{\mu}+2 \nabla^{\mu} E_{\mu \nu}^{\alpha \beta}\left(g^{-1} \delta g\right)_{\beta}^{\nu},
$$

where the rank-4 tensor $E_{\mu \nu}^{\alpha \beta}$ is defined by

$$
E_{\mu \nu}^{\alpha \beta}=\frac{\partial \mathcal{L}}{\partial R_{\alpha \beta}^{\mu \nu}}
$$

For Lagrangians of the form (1), this functional derivative takes the form

$$
E_{\mu \nu}^{\alpha \beta}=\frac{1}{2}\left(\delta_{[\mu \nu]}^{[\alpha \beta]}+\alpha R_{[\mu}^{[\alpha} \delta_{\nu]}^{\beta \beta}+2 \beta R \delta_{[\mu \nu]}^{[\alpha \beta]}\right),
$$

where $\delta_{[\mu \nu]}^{[\alpha \beta]}$ stands for the totally antisymmetric product of Kronecker tensors with a normalization factor equal to unity.

The vacuum states of the theory correspond to maximally symmetric spaces,

$$
R_{\mu \nu}^{\alpha \beta}=-\frac{1}{\ell^{2}} \delta_{[\mu \nu]}^{[\alpha \beta]},
$$

where $\ell$ is the curvature radius of the space. In four dimensions, the addition of quadratic terms does not alter the relation between $\Lambda$ and $\ell$, which remains $\Lambda=-3 / \ell^{2}$, independent of $\alpha$ and $\beta$. Besides, four dimensions is also special in the sense that all Einstein spacetimes (i.e., $R_{\mu \nu}=-3 / \ell^{2} g_{\mu \nu}$ ) persist as solutions of the theory. 
Notice that for QCG, the second contribution on the righthand side of Eq. (6) vanishes identically for Einstein spaces.

The particle content of the theory can be obtained from the linearization of the field equations (3)-(5) around the AdS background metric $\bar{g}_{\mu \nu}$. By writing $g_{\mu \nu}=\bar{g}_{\mu \nu}+h_{\mu \nu}$, where $h_{\mu \nu}$ is a small perturbation [3], the linearized equations of motion read

$$
\begin{aligned}
\delta\left(G_{\mu \nu}+E_{\mu \nu}\right)= & {[1+2 \Lambda(\alpha+4 \beta)] G_{\mu \nu}^{L} } \\
& +\alpha\left[\left(\bar{\square}-\frac{2 \Lambda}{3}\right) G_{\mu \nu}^{L}-\frac{2 \Lambda}{3} R^{L} \bar{g}_{\mu \nu}\right] \\
& +(\alpha+2 \beta)\left(-\bar{\nabla}_{\mu} \bar{\nabla}_{\nu}+\bar{g}_{\mu \nu} \bar{\square}+\Lambda \bar{g}_{\mu \nu}\right) R^{L},
\end{aligned}
$$

where $G_{\mu \nu}^{L}$ and $R^{L}$ are the linearized versions of the Einstein tensor and Ricci scalar, respectively. The bar denotes a covariant derivative with respect to the background metric, $\bar{g}_{\mu \nu}$.

In order to simplify the analysis of the linearized equations, it is convenient to choose the transversetraceless gauge $\bar{\nabla}_{\mu} h^{\mu \nu}=0$. Then, from the trace of Eq. (10) one gets the equation for the propagation of the scalar mode, namely,

$$
h-2(\alpha+3 \beta) \bar{\square} h=0,
$$

where $h=\bar{g}^{\mu \nu} h_{\mu \nu}$ is the trace of the perturbation, which describes the scalar particle of the theory. For the special choice $\alpha=-3 \beta$, this degree of freedom becomes strongly coupled and disappears from the spectrum.

\section{ENERGY DEFINITION IN QCG}

\section{A. Linearized perturbations and charges}

A definition of gravitational energy for generic QCG theories was given by Deser and Tekin in Refs. $[3,4]$ by extending the work of Abbott and Deser, who had achieved in Ref. [5] such an energy definition for Einstein's theory in the presence of a cosmological constant. This method is thus often referred to as the Abbott-Deser-Tekin formalism, or simply the ADT method. The procedure is based on the linearization of the EOMs around a globally (A)dS (background) metric. The method is also valid in the case of a vanishing cosmological constant. The linearized charges obtained in this way are expressed as the integral over an orientable codimension-2 surface $\Sigma$,

$$
Q_{\mathrm{ADT}}^{\alpha}[\bar{\xi}]=\frac{1}{8 \pi G} \int_{\Sigma} d S_{\beta} q_{\mathrm{ADT}}^{\alpha \beta}
$$

where $d S_{\nu}$ is the differential area element defined by the exterior unit vector normal to the surface and where $\Sigma$ defines the integration at a fixed time and fixed radial coordinate. The integrand is given by the components of the prepotential

$$
\begin{aligned}
q_{\mathrm{ADT}}^{\alpha \beta}= & {[1+2 \Lambda(\alpha+4 \beta)]\left(\bar{\xi}_{\lambda} \bar{\nabla}^{[\alpha} h^{\beta] \lambda}+\bar{\xi}^{[\alpha} \bar{\nabla}^{\beta]} h\right.} \\
& \left.+h^{\lambda[\alpha} \bar{\nabla}^{\beta]} \bar{\xi}_{\lambda}-\bar{\xi}^{[\alpha} \bar{\nabla}_{\lambda} h^{\beta] \lambda}+\frac{1}{2} h \bar{\nabla}^{\alpha \bar{\xi}^{\beta}}\right) \\
& +(\alpha+2 \beta)\left(2 \bar{\xi}^{[\alpha} \bar{\nabla}^{\beta]} R^{L}+R^{L} \bar{\nabla}^{\alpha} \bar{\xi}^{\beta}\right) \\
& -\alpha\left(2 \bar{\xi}_{\lambda} \bar{\nabla}^{[\alpha} G_{L}^{\beta] \lambda}+2 G_{L}^{\lambda[\alpha} \bar{\nabla}^{\beta]} \bar{\xi}_{\lambda}\right),
\end{aligned}
$$

where $\bar{\xi}_{\lambda}$ are the components of the Killing vector that generates the isometry associated with the Noether charge that is being computed. As before, the subscript $L$ stands for the linearized version of every curvature tensor involved.

The ADT method has been shown to be useful to compute the Noether charges associated with several solutions of physical importance. In the case of a Schwarzschild-AdS black hole, for example, whose metric in standard Schwarzschild coordinates is given by

$$
\begin{aligned}
d s^{2}= & -\left(1-\frac{2 m G}{r}+\frac{r^{2}}{\ell^{2}}\right) d t^{2}+\left(1-\frac{2 m G}{r}+\frac{r^{2}}{\ell^{2}}\right)^{-1} d r \\
& +r^{2}\left(\sin ^{2} \theta d \phi^{2}+d \theta^{2}\right),
\end{aligned}
$$

with $t \in \mathbb{R}, \quad r \in \mathbb{R}_{\geq 0}, \phi \in[0,2 \pi], \theta \in[0, \pi]$, the ADT gravitational energy computation yields

$$
M=Q_{\mathrm{ADT}}^{0}\left[\partial_{t}\right]=m[1+2 \Lambda(\alpha+4 \beta)],
$$

which, in particular, tends to the usual mass parameter $m$ in the absence of higher-curvature terms $(\alpha=\beta=0)$ or in the absence of a cosmological constant $(\Lambda=0)$. In general, there is no need to go beyond linear order in the curvature, as the energy is linear in $m$. Thus, even if the Lagrangian contains higher powers in the curvature, the conserved charge can be consistently truncated to an expression linear in $R$ if the corresponding background is not degenerate. An exception to this happens at the so-called critical points, which are special points of the space of coupling constants where the massive spin-2 field actually becomes massless and the spin-0 mode becomes strongly coupled. At such a critical point, the black hole solutions of the QCG theory have vanishing energy and vanishing angular momentum, and the fourth-order linearized equations become confluent and develop low-decaying extra modes. This is, for instance, the case of critical gravity [6], which is defined by choosing the values $\alpha=-3 \beta=3 /(2 \Lambda)$ in Eq. (1). We have already mentioned that when $\alpha=-3 \beta$ the scalar mode in Eq. (11) is eliminated and $h=0$ becomes the only possible solution. Then, Eq. (10) reduces to 


$$
\left(\bar{\square}-\frac{2 \Lambda}{3}\right)\left(\bar{\square}-\frac{2 \Lambda}{3}-\frac{2 \Lambda \beta+1}{3 \beta}\right) h_{\mu \nu}=0,
$$

which describes the propagation of a massless graviton and a massive spin-2 mode. If, in addition, one imposes the particular value $\beta=-1 /(2 \Lambda)$, then the graviton degenerates with the additional spin- 2 mode. Under these conditions, the formula (15) for the mass of the AdS-Schwarzschild black holes yields a vanishing result [6]. This result, however, has to be confirmed by going to next-to-leading order in perturbation theory as, because of the degeneracy in the spin-2 fields, QCG theory at the critical point may present linear instabilities [7] and thus the linear analysis cannot be fully trusted. This is, indeed, one of the main motivations we have to look for an intrinsically nonlinear method to compute conserved charges in QCG, which we will do in the following section.

\section{B. Diffeomorphic charges}

A preliminary ingredient for our discussion is the analysis of the Noether currents carried out by Wald and collaborators for the theory of gravity. Assuming the Lagrangian to be a function of the metric and the curvature tensor, $\mathcal{L}\left(g_{\mu \nu}, R_{\mu \nu \alpha \beta}\right)$, the corresponding Noether current adopts the form

$$
J^{\alpha}=2 E_{\mu \nu}^{\alpha \beta} g^{\nu \lambda} \delta_{\xi} \Gamma_{\beta \lambda}^{\mu}+2 \nabla^{\mu} E_{\mu \nu}^{\alpha \beta}\left(g^{\nu \lambda} \delta_{\xi} g_{\lambda \beta}\right)+\mathcal{L} \xi^{\alpha},
$$

where $\delta_{\xi}$ stands for the Lie derivative acting on the fields,

$$
\begin{aligned}
\delta_{\xi} \Gamma_{\beta \lambda}^{\mu} & =-\frac{1}{2}\left(\nabla_{\beta} \nabla_{\lambda} \xi^{\mu}+\nabla_{\lambda} \nabla_{\beta} \xi^{\mu}\right)-R_{\lambda \sigma \beta}^{\mu} \xi^{\sigma}, \\
\delta_{\xi} g_{\lambda \beta} & =-\left(\nabla_{\lambda} \xi_{\beta}+\nabla_{\beta} \xi_{\lambda}\right) .
\end{aligned}
$$

In contrast to the original derivation in Refs. [8,9], here we will not take the Killing vector condition for the diffeomorphism $\xi^{\mu}$ at this step. This allows us to account for contributions coming from derivatives of the tensor $E_{\mu \nu}$. After some algebraic manipulation, the Noether current is written down as a total derivative plus the field equations contracted with the vector $\xi[10]$,

$$
J^{\alpha}=2 \nabla_{\beta}\left(E_{\mu \nu}^{\alpha \beta} \nabla^{\mu} \xi^{\nu}+2 \nabla^{\mu} E_{\mu \nu}^{\alpha \beta} \xi^{\nu}\right)+(\mathrm{EOM})^{\alpha \beta} \xi_{\beta} .
$$

The above equation implies that the conserved charge associated with an isometry generated by a Killing vector $\xi^{\mu}$ is an integral over a codimension-2 surface $\Sigma$,

$$
Q_{\mathrm{IW}}^{\alpha}[\xi]=\frac{1}{8 \pi G} \int_{\Sigma} d S_{\beta} q_{\mathrm{IW}}^{\alpha \beta}
$$

for the Iyer-Wald prepotential

$$
q_{\mathrm{IW}}^{\alpha \beta}=E_{\mu \nu}^{\alpha \beta} \nabla^{\mu} \xi^{\nu}+2 \nabla^{\mu} E_{\mu \nu}^{\alpha \beta} \xi^{\nu} .
$$

The above formula gives rise to the standard black hole entropy when evaluated at the horizon. However, when it comes to the discussion of charges in the asymptotic region, it is necessary to correct the prepotential as $q_{\mathrm{IW}}^{\alpha \beta} \rightarrow q_{\mathrm{IW}}^{\alpha \beta}+\xi^{[\alpha} B^{\beta]}$, where $B$ is a boundary term whose form can be found by assuming suitable boundary conditions. It is only after the contribution of such a term is added that this construction reproduces the results of the canonical formalism [8].

There exist other methods to compute Noether charges that are suitable to be adapted to the case of Lagrangians with higher-derivative couplings. One particularly strong method is the so-called Barnich-Brandt method [11], which has the advantage of being fully constructive. This method is often used to compute charges associated with asymptotic isometries, including possible central terms in the charge algebra. In the particular case of exact (in opposition to asymptotic) Killing vectors, the method of Ref. [11] agrees with the Iyer-Wald computation, and in the case of black holes it reproduces the ADT charges.

In the following section, we propose a qualitatively different way of regularizing the Iyer-Wald charges for the Lagrangian (1). This amounts to replacing the effect of $B$ by a correction in $q_{\mathrm{IW}}^{\alpha \beta}$ that gives rise to a topological term in the action.

\section{TOPOLOGICAL TERM AND CONSERVED CHARGES}

Commonly omitted from the gravity action in four dimensions, the Pfaffian

$$
\mathcal{E}_{4}=\sqrt{-g}\left(R_{\alpha \beta}^{\mu \nu} R_{\mu \nu}^{\alpha \beta}-4 R^{\mu \nu} R_{\mu \nu}+R^{2}\right)
$$

(hereafter referred to as the Gauss-Bonnet term) can be suitably added to the Lagrangian (1) with a dimensionful coupling $\gamma$. This is indeed a topological term and, as such, it does not affect the dynamics. However, its presence does modify the infrared divergences and the Noether currents. In other words, with the addition of $\gamma \mathcal{E}_{4}$ to $\sqrt{-g} \mathcal{L}$, the bulk field equations $E^{\mu \nu}=0$ do not change, but the surface term does. The latter is augmented by the term

$$
\Theta_{\mathrm{GB}}^{\alpha}=\gamma g^{\nu \lambda} \delta \Gamma_{\beta \lambda}^{\mu} \delta_{[\mu \nu \sigma \lambda]}^{\alpha \alpha \beta \delta]} R_{\gamma \delta}^{\sigma \lambda},
$$

where, again, we are using the totally antisymmetric Kronecker delta symbol as a shorthand. The minimum requirement for Eq. (23) is that the total action attains a well-posed variational principle when evaluated on a vacuum state of the theory. That is, one demands $\delta I_{\text {total }}=0$ on the maximally symmetric solution, namely, global $\mathrm{AdS}_{4}$. The total surface term $\Theta_{\text {total }}^{\alpha}=\Theta_{\mathrm{QCG}}^{\alpha}+\Theta_{\mathrm{GB}}^{\alpha}$ on $\mathrm{AdS}_{4}$ takes the form 


$$
\Theta_{\text {total }}^{\alpha}=\delta_{[\mu \nu]}^{[\alpha \beta]} g^{\nu \lambda} \delta \Gamma_{\beta \lambda}^{\mu}\left[1+2 \Lambda(4 \beta+\alpha)-\frac{4 \gamma}{\ell^{2}}\right],
$$

and demanding that this vanishes amounts to unambiguously setting the coupling of the topological term to

$$
\gamma=\frac{\ell^{2}}{4}[1+2 \Lambda(4 \beta+\alpha)]
$$

This generalizes the result of Ref. [1], to which it reduces when $\alpha=\beta=0$. In other words, Eq. (25) expresses the fact that the coupling constant $\gamma$ includes the effect of the quadratic terms in renormalizing the Newton constant. In the case $\alpha=\beta=0$, Eq. (25) yields $\gamma=-3 /(4 \Lambda)$, which is the precise value that (when the theory is formulated in five dimensions) the coupling constant $\gamma$ has to take for the Einstein-Gauss-Bonnet action to admit to be written as a Chern-Simons gauge theory.

Besides the condition on the vacuum state $\Theta_{\text {total }}^{\alpha}=0$ for the variation of the action to vanish on $\mathrm{AdS}_{4}$, what is not a priori clear is whether or not such a value of $\gamma$ is also the one that produces a finite variation of the action when one departs from the global AdS space and instead considers, for instance, massive solutions. Below, we will show for physically relevant solutions that the addition of the Gauss-Bonnet term (22) to Eq. (25) actually suffices to render finite the energy definition coming from Noether's theorem.

Indeed, once the topological term is taken into account, the prepotential (21) turns into

$$
\begin{aligned}
q_{\mathrm{top}}^{\alpha \beta}= & \frac{1}{2} \nabla^{\mu} \xi^{\nu}\left[\delta_{[\mu \nu]}^{[\alpha \beta]}+\alpha R_{[\mu}^{[\alpha} \delta_{\nu]}^{\beta]}+2 \beta R \delta_{[\mu \nu]}^{[\alpha \beta]}\right. \\
& \left.+\frac{\ell^{2}}{4}(1+2 \Lambda(4 \beta+\alpha)) \delta_{[\mu \nu \sigma \lambda]}^{[\alpha \beta \gamma \delta]} R_{\gamma \delta}^{\sigma \lambda}\right] \\
& +\nabla^{\mu}\left(\alpha R_{[\mu}^{[\alpha} \delta_{\nu]}^{\beta]}+2 \beta R \delta_{[\mu \nu]}^{[\alpha \beta]}\right) \xi^{\nu},
\end{aligned}
$$

and the associated charge (20) for the quadratic-curvature gravity action is expressed as a surface integral,

$$
Q_{\mathrm{top}}^{\alpha}[\xi]=\frac{1}{8 \pi G} \int_{\Sigma} d S_{\beta} q_{\mathrm{top}}^{\alpha \beta}
$$

The subscript "top" refers to the topological origin of the supplementary term (22).

\section{A. Black hole gravitational energy}

Restricting ourselves first to the Einstein sector of the theory, it is clear that the terms containing derivatives of the curvatures in Eqs. (26) and (27) vanish identically. In a similar way, it is easy to notice that the mass is coming only from the Gauss-Bonnet contribution to the charge, which is proportional to the Riemann tensor. This is remarkable, as it manifestly shows that, in this framework, the topological term gathers the relevant physical information. The Ricci tensor and Ricci scalar terms on-shell contribute just with constants to the squared bracket in the relation above.

For the timelike Killing vector $\xi=\partial_{t}$, the charge (26)-(27) gives the mass of Schwarzschild-AdS black holes,

$$
Q_{\mathrm{top}}^{0}\left[\partial_{t}\right]=m[1+2 \Lambda(\alpha+4 \beta)]
$$

which is in exact agreement with the result (15) obtained using the ADT method. A similar result holds for the angular momentum of Kerr-AdS black holes.

The fact that we are able to recover the same expression (15) from a procedure that is intrinsically nonperturbative may shed light on the general problem of linear instability of QCG at critical points. In particular, in the case of critical gravity, the above formula implies the exact vanishing of all conserved quantities for any Einstein space [12].

The contributions of the higher-curvature terms, including the topological term (22), also produce a change in the Bekenstein-Hawking entropy of the black holes. Computed as a charge at the horizon, the entropy gets modified in two ways: apart from the modification of the prefactor $1 /(4 G)$ in the area law, which in the presence of $R^{2}$ terms gets multiplied by a factor $[1+2 \Lambda(\alpha+4 \beta)]$ in such a way that the first principle of black hole mechanics still holds, the presence of the topological term induces an extra shift of the entropy formula, which receives an extra constant piece $4 \pi \gamma / G$. The latter, being a constant, does not affect the first principle.

\section{B. Gravitational waves}

Now, let us focus on other solutions of the theory. Consider non-Einstein spaces that solve the EOM and represent gravitational waves. These are given by a metric of the form [13]

$d s^{2}=\frac{\ell^{2}}{z^{2}}\left[-(1+F(t, z, x)) d t^{2}+2 d t d u+d z^{2}+d x^{2}\right]$,

where $F$ is a function that does not depend on the lightlike coordinate $u$. The coordinates can be taken as $u \in \mathbb{R}$, $t \in \mathbb{R}, z \in \mathbb{R}_{>0}$, and $x \in \mathbb{R}$. The function $F$ can be thought of as describing the profile of the gravitational wave. $F=$ const corresponds to $\mathrm{AdS}_{4}$ spacetime in Poincaré coordinates, with the boundary being located at $z=0$. Actually, $z$ is related to the standard radial coordinate of $\mathrm{AdS}_{4}$ by $z=\ell^{2} / r$.

For a metric of the form (29), the field equations reduce to

$$
\left(\square-\tilde{m}^{2}\right) \square F=0,
$$

with the effective mass parameter 


$$
\tilde{m}^{2}=\frac{6(\alpha+4 \beta)-\ell^{2}}{\alpha} .
$$

A detailed analysis of the geometries (30) was given in Ref. [14], where two sectors of solutions were seen to appear. The functions that solve the second-order equation $\square F=0$ in Eq. (30) correspond to the cases $F(z)=z^{3}$ and $F=$ const. These are solutions of Einstein type and describe the propagation of a massless mode on $\mathrm{AdS}_{4}$. In addition, the theory contains non-Einstein solutions of the form $F(z)=z^{k}$, with

$$
k_{ \pm}=\frac{3}{2} \pm \sqrt{\frac{9}{4}+\frac{6(\alpha+4 \beta)-\ell^{2}}{\alpha}},
$$

which are actually solutions to $\left(\square-\tilde{m}^{2}\right) F=0$. The latter correspond to exact gravitational-wave solutions that propagate on $\mathrm{AdS}_{4}$ as if they have an inertial mass given by Eq. (31). The above values apply to the propagation of massive AdS waves. Because $k_{+}>k_{-}$, only solutions of the form $F(z)=z^{k_{+}}$can be asymptotically, locally $\mathrm{AdS}_{4}$. The condition for this to happen is $8(\alpha+3 \beta) \geq \ell^{2}$. If this is obeyed, then a natural question is whether the gravitational energy associated with these stationary waves on $\mathrm{AdS}_{4}$ is actually finite. Remarkably enough, using the ADT method one obtains that the energy associated with such solutions, considering the configuration $F=0$ as the background, yields a vanishing result, $Q_{\mathrm{ADT}}^{0}\left[\partial_{t}\right]=0$. This may seem puzzling as the value $\tilde{m}^{2} \neq 0$ endows the wave solution with inertial effective mass. Still, the net gravitational ADT energy is zero. This phenomenon is analogous to what has been observed with evanescent gravitons in warped-AdS spaces. In order to check the consistency of our method with the ADT formalism, we should be able to show that Eqs. (26) and (27) also yields a vanishing result.

The computation of the total energy of the massive AdS wave solution $F(z)=z^{k_{+}}$using Eqs. (26) and (27) yields the following result for the prepotential:

$q_{\mathrm{top}}^{\alpha \beta}=z^{k+1} \ell^{-4} k\left(\alpha k^{2}-3 \alpha k+\ell^{2}-6 \alpha-24 \beta\right) \delta_{[u z]}^{[\alpha \beta]}$,

which precisely vanishes when $k=k_{ \pm}$. Therefore, the gravitational energy associated with the solutions $F(z)=z^{k_{+}}$computed with Eqs. (26) and (27) turns out to be zero as well, namely, $Q_{\text {top }}^{0}\left[\partial_{t}\right]=0$. This shows the agreement with the ADT method in the case of a nonEinstein solution of the QCG theory.

\section{Logarithmic modes}

One of the special features that the higher-curvature theories exhibit at the critical points is the emergence of low-decaying modes. These are described by asymptotically AdS solutions with a logarithmic falloff in the radial direction. Such modes appear, for example, in the chiral point of topologically massive gravity [15] and they were also observed in critical gravity in four dimensions and higher [16]. Logarithmic modes in asymptotically AdS space are a symptom of nonunitarity in the dual conformal field theory.

There exist exact solutions to higher-curvature gravity exhibiting logarithmic decay $[17,18]$. These are given by gravitational waves of the type (29) with a profile function $F(r) \propto \log r$. Such modes are present, for example, when the coupling constants in the action obey the special relation

$$
6(\alpha+4 \beta)=\ell^{2}
$$

with $\Lambda=-3 / \ell^{2}$. In particular, this includes critical gravity [6], whose coupling constants satisfy $\alpha=-3 \beta=3 /(2 \Lambda)$; see Ref. [19] for a general description. When Eq. (34) holds, the profile function $F(r)$ of the metrics (29) takes the form $F(r)=c_{0}+c_{1} \log r$, where $c_{0}$ and $c_{1}$ are two arbitrary constants. These modes, when $c_{1} \neq 0$, correspond to non-Einstein spaces that solve the higher-curvature equations of motion with a negative cosmological constant. Since these logarithmic modes decay slowly in asymptotically AdS spaces, it is natural to ask whether this makes the energy formula diverge. Remarkably enough, a direct evaluation of the expression $q_{\text {top }}^{\alpha \beta}$ for the logarithmic solution shows that the prepotential identically vanishes by virtue of Eq. (34). Therefore, as it happens with the gravitational waves discussed in the previous subsection, the computation of gravitational energy associated with the logarithmic solutions is actually zero, despite the weakened asymptotics.

\section{Other asymptotics}

There exist other non-Einstein solutions to QCG which describe black holes with different asymptotics. At large distances, such solutions tend to spaces that are not locally $\mathrm{AdS}_{4}$ but have fewer isometries. An example whose metric is known analytically is the Lifshitz black hole solution [20],

$$
\begin{aligned}
d s^{2}= & -\frac{r^{2 z}}{\ell^{2 z}}\left(1-\frac{r_{H}^{n}}{r^{n}}\right) d t^{2}+\frac{\ell^{2}}{r^{2}}\left(1-\frac{r_{H}^{n}}{r^{n}}\right)^{-1} d r^{2} \\
& +\frac{r^{2}}{\ell^{2}}\left(d x^{2}+d y^{2}\right),
\end{aligned}
$$

where $r_{H}$ is an integration constant that gives the location of the horizon and $\ell^{2}$ is the curvature radius fixed in terms of the cosmological constant $\Lambda$ (see below). $z$ is the socalled dynamical exponent (typically $z \geq 1$ ), and $n$ is a real number (we assume $n \geq 2$ ). Coordinates are taken to be $t \in \mathbb{R}, r \in \mathbb{R}_{\geq 0}, x \in \mathbb{R}, y \in \mathbb{R}$. 
The metric (35) plays an important role in the holographic description of nonrelativistic condensed matter systems. Asymptotically, Eq. (35) enjoys anisotropic scale invariance under the transformation $t \rightarrow \lambda^{z} t, r \rightarrow \lambda^{-1} r$, $x \rightarrow \lambda x, y \rightarrow \lambda y$, which comes to realize geometrically the symmetry that the so-called fixed Lifshitz points exhibit [21]. The presence of a horizon at $r=r_{H}$ introduces finite temperature into the problem.

Remarkably, the metric (35) is a solution to the QCG theory [20] provided $\alpha=0$ and $\beta=-1 /(8 \Lambda)=\ell^{2} / 33$, and for the specific value $z=3 / 2$ of the dynamical exponent and $n=3$.

In order to use our method to compute the gravitational energy associated with Eq. (35), we need to set the value of the couplings constant of the topological term, $\gamma$. As for the case of asymptotically $\mathrm{AdS}_{4}$ spaces, we can fix the value of $\gamma$ by requiring the variation of the action on the background geometry to vanish. In the case of a Lifshitz black hole, the background geometry corresponds to the global Lifshitz space with $r_{H}=0$. The latter geometry is singular as it contains incomplete timelike geodesics. However, all of the curvature invariants associated with it turn out to be constant, and in particular $R=-33 /\left(2 \ell^{2}\right)$. This causes the first term $\left(\frac{1}{2}+\beta R\right) \delta_{\alpha \beta}^{\mu \nu}$ in $E_{\alpha \beta}^{\mu \nu}$ to vanish, giving rise to $E_{\alpha \beta}^{\mu \nu}=\frac{\gamma}{2} \delta_{\alpha \beta \gamma \delta}^{\mu \nu \tau \sigma} R_{\tau \sigma}^{\gamma \delta}$ proportional to $\gamma$, whose divergence vanishes, $\nabla^{\mu} E_{\mu \nu}^{\alpha \beta}=0$. Then, the surface term $\Theta_{\text {total }}^{\alpha}$ simply reduces to

$$
\Theta_{\text {total }}^{\alpha}=\gamma g^{\nu \lambda} \delta \Gamma_{\beta \lambda}^{\mu} \delta_{\mu \nu \sigma \lambda}^{\alpha \beta \gamma \delta} R_{\gamma \delta}^{\sigma \lambda}
$$

which implies $\gamma=0$. Therefore, the prepotential reduces to that of Iyer-Wald, $q_{\mathrm{top}}^{\alpha \beta}=q_{\mathrm{IW}}^{\alpha \beta}=0$, and thus the mass of the Lifshitz black hole (35) is found to be zero, $Q_{\mathrm{top}}^{0}\left[\partial_{t}\right]=0$, in agreement with the result of Ref. [20].

The fact that the prepotential vanishes shows that the solution of Ref. [22] exists at a singular point in the parameter space where the action identically vanishes. It is then natural to ask what is the result for the case of nonEinstein black hole solutions like Eq. (35) but where the action does contribute. It was shown in Ref. [22] that QCG also admits solutions of the type (35) at a different point of the parameter space. More precisely, a black hole solution with $z=6$ and $n=4$ exists provided $\alpha=$ $-(5 / 3)^{2} \beta=(5 \ell / 8)^{2}$ and $\Lambda=-51 /\left(2 \ell^{2}\right)$, and this solution, unlike the one of Ref. [20], has a nonzero action. It can be shown that for such a solution the topological term (22) does not suffice to regularize the action and provide a gravitational energy computation. This manifestly shows that the fact that such a method works well for asymptotically AdS spaces is nontrivial. An underlying reason lies in the conformal properties of asymptotically Einstein spaces, for which the contribution of the topological term turns out to agree with those entering in the so-called Ashtekar-Magnon-Das conformal mass [23,24].
We will not discuss precise relation with the conformal mass here; see Refs. [25,26].

\section{CONCLUSIONS}

Motivated by the problem of linear instabilities of highercurvature gravity and by the importance such corrections to Einstein's theory have in the context of quantum gravity, in this paper we gave a definition of gravitational energy for such a theory including arbitrary quadratic-curvature corrections to Einstein's equations. We focused on the case of four-dimensional theories in the presence of a negative cosmological constant, and with asymptotic AdS boundary conditions. For these theories, we computed the gravitational energy and angular momentum of black holes, obtaining results in perfect agreement with previous computations performed using different methods. Our method, however, is intrinsically nonlinear as it relies on the idea of adding to the gravity action topological invariant terms which suffice to regularize the Noether charges and render the variational problem well-posed. This makes our method well-suited to study the higher-curvature theories at the so-called critical points, where linear instabilities arise. The idea of adding to the gravitational action topological invariants to regularize the Noether charges was previously developed by one of the authors and collaborators in the case of second-order theories, such as general relativity, and here we have shown how this can be extended to higher-derivative theories. In addition to black holes, we discussed other solutions, such as gravitational waves in AdS. This enabled us to probe the consistency of our approach in the non-Einstein sector of the quadratic-curvature theories.

Several open questions remain for future investigations. For instance, it would be interesting to explore the extension of this method to higher-derivative theories in higher (even) dimensions, where the quadratic-curvature corrections introduce new qualitative features. It would also be interesting to investigate this method in the context of AdS/CFT holography, in particular the connection between topological terms and the renormalization of holographic correlation functions calculated for this gravity theory, along the lines of Refs. [27-30]. On general grounds, the study of higher-curvature gravity in the context of AdS/CFT is important as fourth-order field equations introduce new sources at the boundary. It is then required to fully understand whether the addition of a topological invariant always produces a variational principle which is finite and expressible in terms of sources kept fixed at the conformal boundary.

\section{ACKNOWLEDGMENTS}

This work was supported in parts by the NSF through Grant No. PHY-1214302, Chilean FONDECYT Project No. 1170765 and the Grant VRIEA-PUCV No. 039.314/ 2018. D. R. B. is a UNAB M.Sc. Scholarship holder. 
[1] R. Aros, M. Contreras, R. Olea, R. Troncoso, and J. Zanelli, Conserved Charges for Gravity with Locally AdS Asymptotics, Phys. Rev. Lett. 84, 1647 (2000).

[2] K. S. Stelle, Renormalization of higher derivative quantum gravity, Phys. Rev. D 16, 953 (1977).

[3] S. Deser and B. Tekin, Energy in generic higher curvature gravity theories, Phys. Rev. D 67, 084009 (2003).

[4] S. Deser and B. Tekin, Gravitational Energy in Quadratic Curvature Gravities, Phys. Rev. Lett. 89, 101101 (2002).

[5] L.F. Abbott and S. Deser, Stability of gravity with a cosmological constant, Nucl. Phys. B195, 76 (1982).

[6] H. Lu and C. N. Pope, Critical Gravity in Four Dimensions, Phys. Rev. Lett. 106, 181302 (2011).

[7] B. Tekin and E. Altas, Linearization instability for generic gravity in AdS spacetime, Phys. Rev. D 97, 024028 (2018).

[8] V. Iyer and R. M. Wald, Some properties of Noether charge and a proposal for dynamical black hole entropy, Phys. Rev. D 50, 846 (1994).

[9] R. M. Wald and A. Zoupas, General definition of conserved quantities in general relativity and other theories of gravity, Phys. Rev. D 61, 084027 (2000).

[10] H. Maeda, R. Svarc, and J. Podolsky, Decreasing entropy of dynamical black holes in critical gravity, J. High Energy Phys. 06 (2018) 118.

[11] G. Barnich and F. Brandt, Covariant theory of asymptotic symmetries, conservation laws and central charges, Nucl. Phys. B633, 3 (2002).

[12] G. Anastasiou, R. Olea, and D. Rivera-Betancour, NoetherWald energy in critical gravity, arXiv:1707.00341.

[13] J. Podolsky, Interpretation of the Siklos solutions as exact gravitational waves in the anti-de Sitter universe, Classical Quantum Gravity 15, L719 (1998).

[14] E. Ayon-Beato, G. Giribet, and M. Hassaine, Deeper discussion of Schrödinger invariant and logarithmic sectors of higher-curvature gravity, Phys. Rev. D 83, 104033 (2011).

[15] D. Grumiller and N. Johansson, Instability in cosmological topologically massive gravity at the chiral point, J. High Energy Phys. 07 (2008) 134.
[16] M. Porrati and M. M. Roberts, Ghosts of critical gravity, Phys. Rev. D 84, 024013 (2011).

[17] M. Alishahiha and R. Fareghbal, $D$-dimensional log gravity, Phys. Rev. D 83, 084052 (2011).

[18] E. A. Bergshoeff, O. Hohm, J. Rosseel, and P. K. Townsend, Modes of log gravity, Phys. Rev. D 83, 104038 (2011).

[19] E. Ayon-Beato, G. Giribet, and M. Hassaine, Critical gravity waves, in Proceedings of the Thirteenth Marcel Grossmann Meeting, edited by K. Rosquist, R. T. Jantzen, and R. Ruffini (World Scientific, Singapore, 2012), p. 1074.

[20] R.-G. Cai, Y. Liu, and Y. W. Sun, A Lifshitz black hole in four dimensional $R^{2}$ gravity, J. High Energy Phys. 10 (2009) 080.

[21] S. Kachru, X. Liu, and M. Mulligan, Gravity duals of Lifshitz-like fixed points, Phys. Rev. D 78, 106005 (2008).

[22] E. Ayon-Beato, A. Garbarz, G. Giribet, and M. Hassaine, Analytic Lifshitz black holes in higher dimensions, J. High Energy Phys. 04 (2010) 030.

[23] A. Ashtekar and A. Magnon, Asymptotically anti-de Sitter space-times, Classical Quantum Gravity 1, L39 (1984).

[24] A. Ashtekar and S. Das, Asymptotically anti-de Sitter space-times: Conserved quantities, Classical Quantum Gravity 17, L17 (2000).

[25] Y. Pang, Brief note on AMD conserved quantities in quadratic curvature theories, Phys. Rev. D 83, 087501 (2011).

[26] R. Araneda, R. Aros, O. Miskovic, and R. Olea, Magnetic mass in 4D AdS gravity, Phys. Rev. D 93, 084022 (2016).

[27] O. Miskovic and R. Olea, Topological regularization and self-duality in four-dimensional anti-de Sitter gravity, Phys. Rev. D 79, 124020 (2009).

[28] O. Miskovic, R. Olea, and M. Tsoukalas, Renormalized AdS action and critical gravity, J. High Energy Phys. 08 (2014) 108.

[29] N. Johansson, A. Naseh, and T. Zojer, Holographic twopoint functions for 4d log-gravity, J. High Energy Phys. 09 (2012) 114.

[30] G. Anastasiou and R. Olea, Holographic correlation functions in critical gravity, J. High Energy Phys. 11 (2017) 019. 